\title{
Initial stage of quasiparticle decay in fermionic systems
}

\author{
Y. Pavlyukh* and J. Berakdar \\ Institut für Physik, Martin-Luther-Universität Halle-Wittenberg, 06120 Halle, Germany
}

\begin{abstract}
A. Rubio
Nano-Bio Spectroscopy Group and ETSF Scientific Development Centre, Dpto. de Física de Materiales, Universidad del País Vasco, CFM CSIC-UPV/EHU-MPC and DIPC, Av. Tolosa 72, E-20018 San Sebastián, Spain
\end{abstract}

(Received 5 November 2010; revised manuscript received 27 December 2012; published 4 March 2013)

\begin{abstract}
Generally, the addition or removal of a single particle in a many-body system does not correspond to an exact eigenstate of the system. Thus the resulting coherent excitation evolves in time. As discussed here, the evolution at short times upon the excitation with the energy $\epsilon$ exhibits a quadratic decay [with the rate constant $\sigma^{2}(\epsilon)$ ]. Later on, after some time $\tau(\epsilon)$, the exponential decay sets in. It is governed by another rate constant $\gamma(\epsilon)$. This behavior is generic for many realistic finite and extended systems. For a finite system it is possible to assess this behavior full numerically using an exact solution of the many-body problem. We present a simple model for the electron spectral function that links together all three aforementioned parameters and give a prescription for how the energy uncertainty $\sigma^{2}(\epsilon)$ can be computed within the many-body perturbation theory. Our numerical results demonstrate that the model approach accurately reproduces the exact spectral function in a large range of energies even in the case of fragmented many-body states. We show that the central quantity of this study $\sigma^{2}(\epsilon)$ can easily be computed exactly or from approximate theories and, hence, can be used for their validation. We also point out how the set in time can be tested by means of attosecond spectroscopy.
\end{abstract}

DOI: 10.1103/PhysRevB.87.125101

PACS number(s): 71.10.Ay, 73.22.Dj

\section{INTRODUCTION}

Undoubtedly, one of the most exciting developments over the last decade has been the development of time-resolved spectroscopic techniques with the attosecond resolution (Refs. 1-3 and references therein). This opens the door to address questions regarding the nature of the formation, evolution, and decay of electronic states which in essence govern the optical, transport, and magnetic properties of matter. For instance, in Ref. 4 it has been shown experimentally for a tungsten surface that the photoelectrons emitted from a core level emerge with a time delay of $\approx 80$ as with respect to those leaving the conduction band. Conceptually similar experiments on $\mathrm{Ne}$ report a time delay of $21 \pm 5$ as between the emission of the $2 s$ and $2 p$ orbitals. ${ }^{5}$ In view of these experiments we address here the fundamental questions of (i) how fast an excitation decays following removal or addition of a particle to the system, (ii) what governs this decay, and (iii) whether we can extract some information about the system upon measuring the decay rate via attosecond spectroscopy.

Our aim is to deliver precise analysis and predictions based on a full numerical solution of the many-body problem which is currently feasible for finite systems. For a qualitative understanding let us recall at first the statements that can be made for extended systems based on Landau's theory of Fermi liquids ${ }^{6}$ which describes (low-energy) long-lived excitations as quasiparticles (QPs). Generally, QPs decay in time due to residual interactions. How this decay proceeds in time $t$ is known under certain conditions only: A QP decays exponentially as $\sim \exp (-\gamma t)$ with the rate constant $\gamma(\epsilon) \sim \epsilon^{2} / \epsilon_{F}{ }^{7}$ Here $\epsilon$ is the quasiparticle energy and $\epsilon_{F}$ is the Fermi energy. This, however, holds true only for times $t \gg 1 / \gamma$, which excludes the set-in regime right after the excitation. The importance of this restriction is illustrated by the following: Let us assume an exponential decay at all $t$, thus the QP peak appears in frequency with a Lorentzian shape. This means that, at the QP energy $\epsilon$, the spectral function behaves as

$$
A_{\mathrm{L}}(\omega ; \epsilon)=\frac{1}{\pi} \frac{\gamma}{(\omega-\epsilon)^{2}+\gamma^{2}} .
$$

The standard deviation $\sigma^{2}(\epsilon)$ of the spectral density given by such a functional form diverges,

$$
\sigma^{2}(\epsilon)=\int_{-\infty}^{\infty} d \omega(\omega-\epsilon)^{2} A_{\mathrm{L}}(\omega ; \epsilon) \rightarrow \infty .
$$

Explicit calculations for a three-dimensional (3D) homogeneous electron gas (HEG) show that this divergence is spurious $^{8}$ and that the zero, first, and second spectral moments are indeed finite. The convergence of the integral Eq. (1) is governed by the high-frequency behavior of the spectral function. Thus, the short-time limit of the single-particle Green's function (from which $A_{\mathrm{L}}$ derives) is of a particular interest. Quantum-kinetics indicates a quadratic decay in time. ${ }^{9}$ It is conceivable that the above arguments can be repeated for states appearing with a Lorentzian spectral shape, even for few-body systems. Hence, measuring the initial time evolution, i.e., right after one electron has been removed or added, yields information beyond the spectral distribution of the states. It is our goal to quantify that information in terms of physical processes and to offer a scheme for their computation. As an example we perform calculations for small metal clusters.

\section{SPECTRAL FUNCTION}

The spectral function of a fermionic many-body quantum system is defined in its most general form as the overlap of a 
particle and hole states:

$$
S_{i j}\left(t-t^{\prime}\right)=\frac{1}{2 \pi}\left[\left\langle H_{i}(t) \mid H_{j}\left(t^{\prime}\right)\right\rangle+\left\langle P_{i}(t) \mid P_{j}\left(t^{\prime}\right)\right\rangle\right] .
$$

The states emerging by adding $\left.\left[\left|P_{i}(t)\right\rangle=\left|\hat{\psi}_{i}^{\dagger}(t)\right| \mathrm{GS}\right\rangle\right]$ or removing $\left.\left[\left|H_{i}(t)\right\rangle=\left|\hat{\psi}_{i}(t)\right| \mathrm{GS}\right\rangle\right]$ a particle in the ground state are not the eigenstates of the system in general and, thus, decay in time. Typically, the single-particle basis can be constructed so that the matrix (2) is nearly diagonal (for homogeneous translationally-invariant systems it is true in general) and we can label its elements by the corresponding state energies. Furthermore, it is convenient to represent the spectral function as a product of a noninteracting oscillatory part and a decaying part $A(t ; \epsilon)$ :

$$
S(t ; \epsilon)=\frac{e^{-i \epsilon t}}{2 \pi} A(t ; \epsilon) .
$$

While the exponential decay in the long-time limit,

$$
A(t ; \epsilon) \stackrel{t \rightarrow \infty}{\longrightarrow} e^{-\gamma t},
$$

is a renown feature of Fermi liquids there is no evidence for a universal short-time limit. In general, $A(t ; \epsilon)$ is a complicated nonanalytic function in the vicinity of $t=0$. However, these complications can only arise as a result of integration over infinitely many scattering channels of the quasiparticle decay. In finite systems, or when a quasiparticle decays due to the interaction with a bounded spectrum of bosonic excitations such as longitudinal optical phonons ${ }^{10}$ or plasmons $^{11}$ the function is well behaved. The lowest-order cumulant expansion for the Green's function leads then to the quadratic decay

$$
\frac{d}{d t} A(t ; \epsilon) \stackrel{t \rightarrow 0}{\longrightarrow}-\sigma^{2}(\epsilon) t .
$$

In the present work we propose a model spectral function that exhibits the correct short- and long-time behavior, demonstrate how its parameters can be computed based on many-body perturbation theory, and present numerical results for a finite system as an illustration of the accuracy of the theory and as a justification of further approximations. An attempt with a similar goal has been undertaken in Ref. 10; the resulting spectral function, however, violates the sum rules and has a shape with the spectral moments finite at any order-at variance with Ref. 8 . The spectral function given in this work fulfills all sum rules and complies with the exact short- and long time-limits. In fact, in a recent work ${ }^{12}$ we discussed and justified this ansatz for a homogenous electron gas. The key ingredients are the imaginary part of the on-shell electron self-energy $\gamma(\epsilon)=\operatorname{Im} \Sigma(\omega=\epsilon ; \epsilon)$ and the decay constant $\sigma^{2}(\epsilon)$ as expressed in terms of the zeroth spectral moment of the self-energy $\Sigma^{(0)}=\frac{1}{\pi} \int_{-\infty}^{\infty} d \omega|\operatorname{Im} \Sigma(\omega ; \epsilon)|$. The approach provides a recipe to compute the short-time limit of the electron correlation function on the basis of many-particle perturbation theory. In particular, we demonstrate how the decay constant [Eq. (5)] can be computed diagrammatically to any desired order in the interaction. Conceptually, the problem should be addressed by quantum kinetic theory. However, in this formalism, analytic calculations of the initial stage of the quasiparticle are not available and numerical approaches rely on further approximations. ${ }^{13-15}$

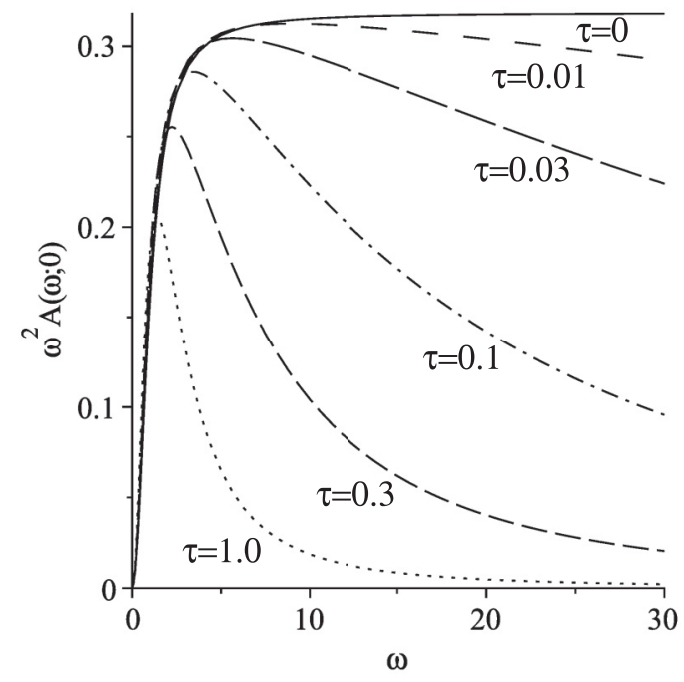

FIG. 1. $\omega^{2} A(\omega ; 0)$ for model spectral function defined by Eq. (6) for rate constant $\gamma=1.0$ and different values of set-in time.

\section{MODEL SPECTRAL FUNCTION}

By the definition (2), the spectral function fulfills $S^{*}(t ; \epsilon)=$ $S(-t ; \epsilon)$ and thus in the frequency domain $S(\omega ; \epsilon)$ is real and positive. For the decaying part (3) we make an ansatz

$$
A(t ; \epsilon)=\exp \left(-\gamma(\epsilon) \frac{t^{2}}{t+\tau(\epsilon)}\right) \text { for } t>0,
$$

which obeys the two limiting cases [Eqs. (5) and (4)] with $\sigma^{2}(\epsilon)=2 \gamma / \tau$. The Fourier transform of the spectral function can be written as follows:

$A(\omega ; \epsilon)=\int_{-\infty}^{\infty} d t e^{i \omega t} S(t ; \epsilon)=\frac{1}{\pi} \int_{0}^{\infty} \cos [(\omega-\epsilon) t] A(t ; \epsilon) d t$.

For $A(t ; \epsilon)$ given by Eq. (6) the analytic form of the Fourier transform is not known, but can easily be obtained numerically (Fig. 1) and it is also possible to prove its positivity. ${ }^{16,17}$ Its odd spectral moments are zero because of symmetry consideration $A(t ; \epsilon)=A(-t ; \epsilon)$ :

$$
\bar{M}^{(2 k-1)}(\epsilon)=\int_{-\infty}^{\infty} d \omega(\omega-\epsilon)^{2 k-1} A(\omega ; \epsilon)=0 .
$$

Thus $\bar{M}^{(1)}(\epsilon)=M^{(1)}(\epsilon)-\epsilon=0$. The even spectral moments can be obtained analytically from the derivatives of $A(t ; \epsilon)$ at $t=0$ :

$\bar{M}^{(2 k)}(\epsilon)=\int_{-\infty}^{\infty} d \omega(\omega-\epsilon)^{2 k} A(\omega ; \epsilon)=(-1)^{k} \lim _{t \rightarrow 0} A^{(2 k)}(t ; \epsilon)$.

This leads to $\bar{M}^{(0)}(\epsilon)=M^{(0)}(\epsilon)=1$ (normalization condition) and $\bar{M}^{(2)}(\epsilon)=M^{(2)}(\epsilon)-\left[M^{1}(\epsilon)\right]^{2}=\frac{2 \gamma(\epsilon)}{\tau(\epsilon)}$. Higher spectral moments diverge because of the discontinuity of the derivative of $A(t ; \epsilon)$ at $t=0$. This completes the proof that in the frequency domain it has exactly three finite spectral moments in accordance with Ref. 8. The asymptotic large- $\omega$ expansion can be obtained by integrating Eq. (7) by parts:

$$
A(\omega ; \epsilon) \sim \frac{6}{\pi} \frac{\gamma}{(\omega-\epsilon)^{4} \tau^{2}} \text { as } \omega \rightarrow \infty .
$$


According to Altshuler et al. ${ }^{18}$ the initial stage of the quasiparticle decay involves a formation of the two-particleone-hole state $(2 p 1 h)$. The rate of the process is given by the first collision time $1 / \tau_{1}$ and is determined by the corresponding Coulomb matrix elements or, in other words, by the available phase space (the energy and momentum must be conserved). The phase space also determines in a crucial way the subsequent stages of the QP decay, which results in the creation of an increasing number of particles and holes, forming either localized or delocalized states in a Fock space. In the latter scenario the exponential decay is established after many generations of particles and holes have emerged. From these very general arguments it is obvious that the exponential decay requires a certain time to develop, which in our theory is determined by the parameter $\tau(\epsilon)$. This time parameter certainly exceeds the first collision time $\left[\tau(\epsilon) \gg \tau_{1}\right]$ obtained from the golden rule arguments applied to the bare Coulomb interaction (at the initial stages the screening is not efficient). This indicates that the time $\tau(\epsilon)$ can be obtained from neither the bare nor the screened interaction and is distinct from the relaxation time at the large-time limit $[1 / \gamma(\epsilon)]$.

To obtain $\tau(\epsilon)$ let us recall the relations between the $n$ th-order spectral moments $\mathbf{M}^{(n)}$ of the single-particle Green function and that of the self-energy $\boldsymbol{\Sigma}^{(n)}$ (Ref. 8):

$$
\begin{gathered}
\mathbf{M}^{(0)}=\mathbf{I}, \quad \delta \boldsymbol{\Sigma}_{\infty}=\mathbf{M}^{(1)}-\boldsymbol{\varepsilon}, \\
\boldsymbol{\Sigma}^{(0)}=\mathbf{M}^{(2)}-\left[\mathbf{M}^{(1)}\right]^{2} .
\end{gathered}
$$

$\boldsymbol{\Sigma}_{\infty}=\delta \boldsymbol{\Sigma}_{\infty}+\boldsymbol{\Sigma}_{\infty}^{0}$ is the frequency-independent real part of the self-energy. ${ }^{19}$ These matrix relations directly follow from the Dyson equation and can be obtained in any basis (Ref. 8 used a plane-waves basis). $\varepsilon$ is a diagonal matrix with the elements given by the zeroth-order-state energies (they already contain mean-field Coulomb interaction and, therefore, include $\Sigma_{\infty}^{0}$; see Appendix B for exact definitions). For finite systems, Hartree-Fock (HF) basis states seems to be appropriate. Writing the matrix of the spectral functions in terms of the imaginary part of the single-particle Green function $\left[\mathbf{A}(\omega)=\frac{1}{\pi}|\operatorname{Im} \mathbf{G}(\omega)|\right]$ and likewise for the spectral function of the self-energy $\left[\mathbf{S}(\omega)=\frac{1}{\pi}|\operatorname{Im} \boldsymbol{\Sigma}(\omega)|\right]$ and using the superconvergence theorem, ${ }^{20}$ the matrices are cast as frequency integrals:

$$
\begin{gathered}
\mathbf{M}^{(n)}=\int_{-\infty}^{\infty} d \omega \omega^{n} \mathbf{A}(\omega), \quad n=0, \ldots, 2, \\
\boldsymbol{\Sigma}^{(0)}=\int_{-\infty}^{\infty} d \omega \mathbf{S}(\omega) .
\end{gathered}
$$

In the HF basis $\delta \boldsymbol{\Sigma}_{\infty}$ is rather small and is proportional to the difference between the direct and the exchange Coulomb energy computed with the Hartree-Fock and exact density matrix, i.e., related to the deviation of the natural occupations from 1 or 0 . Thus, by virtue of Eqs. (8) and (10), we arrive at the conclusion that the Hartree-Fock energies in the first approximation are given by the center of mass of the spectral function. Likewise, by using Eqs. (9) and (10), we establish a formula for the matrix of standard deviations [cf. Eq. (1)]:

$$
\boldsymbol{\sigma}^{2}=\int_{-\infty}^{\infty} d \omega \mathbf{S}(\omega)
$$

Its physical meaning in view of the relation (9) is the energy uncertainty of a many-body state. Formally, an exact representation of this positively defined matrix can be written in terms of the six-point response function ${ }^{21}$ [Fig. 2(a)]:

$$
\begin{aligned}
\Sigma\left(1,1^{\prime}\right)-\Sigma_{\infty}\left(1,1^{\prime}\right) \\
=\int d(23) v(12) \mathcal{G}\left(3^{+} 12,32^{+} 1^{\prime}\right) v\left(31^{\prime}\right) \\
\quad-\int d(2345) v(12) \mathcal{G}\left(12,2^{+} 5\right) \mathcal{G}^{-1}(5,4) \mathcal{G}\left(42^{+}, 21^{\prime}\right) v\left(21^{\prime}\right) \\
\equiv \\
\quad \int d(23) v(12) G^{(3)}\left(3^{+} 12,32^{+} 1^{\prime}\right) v\left(31^{\prime}\right),
\end{aligned}
$$

where $\mathcal{G}\left(1, \ldots n ; 1^{\prime}, \ldots, n^{\prime}\right)$ denotes a general $n$-particle Green's function, and $G^{(3)}\left(123,1^{\prime} 2^{\prime} 3^{\prime}\right)$ stands for the irreducible $2 p 1 h$ Green's function. Its diagrammatic expansion in terms of the bare propagators is such that cutting a single fermionic line in each constituent diagram cannot separate the $2 p 1 h$ entrance and exit channels. ${ }^{22}$ In Appendix A we sketch how the representation (14) can be derived from the equations of motion for the Green's functions. In the present work, we compute the matrix standard deviations (12) for a finite system using different factorizations of $G^{(3)}$. Such an unusual approach to generate approximations for the electron self-energy is motivated by the fact that the configurationinteraction (CI) approach is capable of providing exact $n$-particle correlation functions in contrast to the ordinary many-body perturbation theory which tries to approach them by systematically summing up some classes of diagrams. We judge the accuracy of different approximations for the self-energy by comparing its zeroth spectral moment with exact value from a full CI approach for a finite electron system.

\section{NUMERICAL RESULTS}

As a prototypical system we consider the widely studied $\mathrm{Na}_{9}{ }^{+}$cluster: ${ }^{23-25}$ a small number of electrons makes it accessible to the full CI approach. ${ }^{26,27}$ The $\sigma^{2}$ matrix can be computed exactly by exact diagonalization of the many-body Hamiltonian. We use an algorithm by Olsen et al. ${ }^{28}$ based on the graphical unitary group approach ${ }^{29}$ for the generation of the restricted active space (RAS) and full CI Hamiltonians. The calculations are performed for each spin multiplicity separately using spin-adapted basis functions. ${ }^{30}$ In terms of the matrix elements of the creation and annihilation operators the spectral moments are expressed as

$$
\mathbf{M}^{(n)}=\sum_{p}^{\mathcal{D}^{\mathrm{N}+1}}\left(\varepsilon_{p}^{+}\right)^{n} \mathbf{X}^{p} \overline{\mathbf{X}}^{p}+\sum_{q}^{\mathcal{D}^{\mathrm{N}-1}}\left(\varepsilon_{q}^{-}\right)^{n} \mathbf{Y}^{q} \overline{\mathbf{Y}}^{q},
$$

where the summation is performed over the Hilbert space of the ionized states (dimension $\mathcal{D}^{\mathrm{N}-1}$ ) and electron attached states (dimension $\mathcal{D}^{\mathrm{N}+1}$ ). The matrix elements of electron creation $\left(\hat{a}_{\alpha}^{\dagger}\right)$ and annihilation $\left(\hat{a}_{\alpha}\right)$ operators

$$
X_{\alpha}^{p}=\left\langle p \mathrm{~N}+1\left|\hat{a}_{\alpha}^{\dagger}\right| 0 \mathrm{~N}\right\rangle, \quad Y_{\alpha}^{q}=\left\langle q \mathrm{~N}-1\left|\hat{a}_{\alpha}\right| 0 \mathrm{~N}\right\rangle,
$$

and the transition energies $\varepsilon_{p}^{+}=E_{p}^{\mathrm{N}+1}-E_{0}^{\mathrm{N}}$, $\varepsilon_{q}^{-}=E_{0}^{\mathrm{N}}-E_{q}^{\mathrm{N}-1}$ are computed from the CI many-body states. Formally, for a finite system in a finite basis, Eq. (15) yields nondiverging moments of any order. This is, however, 
(a)

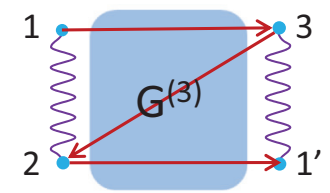

(b)

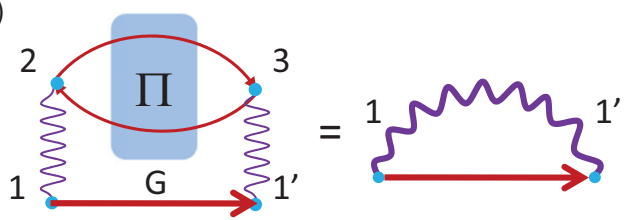

(c)

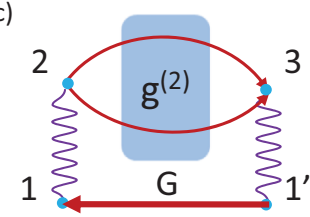

FIG. 2. (Color online) Diagrams for electron self-energy. (a) exact expression in terms of the $2 p 1 h$ response function in which the entrance and exit channels cannot be separated by cutting one fermion line. (b) ( $p-h)$ - $p$ factorization leading to the $G W$ approximation. (c) ( $p$ - $p$ )- $h$ factorization.

an artifact of the numerical approach resulting from the truncation of the Hilbert space.

Approximations for the self-energy operator can be obtained from the factorization of the $2 p 1 h$ six-point function $^{19,21}$ [Fig. 2(a)]. If the particle-hole $(p-h)$ Green's function is treated exactly we obtain the so-called $G W$ approximation $^{31}$ [Fig. 2(b)]. Alternatively, this approximation can be obtained from the $\Psi[\mathcal{G}, \mathcal{W}]$ variational energy functional $^{32}$ expanded in terms of the dressed electron propagator $G$ and the screened Coulomb interaction $\mathcal{W}$. A single diagram of the first order has to be considered. Finally, one obtains the same functional form by neglecting the threepoint vertex function $\Gamma$ in Hedin's equations. ${ }^{33}$ It should be noted that we do not perform the self-consistent solution of Hedin's equations; instead, we compute exactly $\mathcal{G}$ and $\mathcal{W}$ from the exact one-particle and particle-hole propagators. They are given by the Lehmann representations in terms of many-body electron states. ${ }^{34,35}$ In accordance with the spectral representation of the self-energy ${ }^{26}$ we obtain for the energy uncertainty

$$
\Sigma_{\alpha, \beta}^{(0), G W}=4 \sum_{n \neq 0}^{\mathcal{D}^{\mathrm{N}}} \sum_{p}^{\mathcal{D}^{\mathrm{N}+1}}\langle\alpha p \mid n\rangle\langle n \mid p \beta\rangle+(p-h)-h \text { terms },
$$

where we introduced a notation for the convolution of the Coulomb matrix elements $\langle\alpha \beta \mid \gamma \delta\rangle=$ $\int d\left(\mathbf{r}_{1} \mathbf{r}_{2}\right) \phi_{\alpha}\left(\mathbf{r}_{1}\right) \phi_{\beta}\left(\mathbf{r}_{1}\right) \phi_{\gamma}\left(\mathbf{r}_{2}\right) \phi_{\delta}\left(\mathbf{r}_{2}\right) /\left|\mathbf{r}_{1}-\mathbf{r}_{2}\right|$ with matrix elements of the creation (or annihilation) operators [Eq. (15)] and with the density matrix elements $Q_{\gamma \delta}^{n}=\left\langle n \mathrm{~N}\left|\hat{a}_{\gamma}^{\dagger} \hat{a}_{\delta}\right| 0 \mathrm{~N}\right\rangle$ :

$$
\langle\alpha p \mid n\rangle=\sum_{\beta} \sum_{\gamma \delta} X_{\beta}^{p}\langle\alpha \beta \mid \gamma \delta\rangle Q_{\gamma \delta}^{n} .
$$

Analogically, one obtains an expression for the self-energy using the $(p-p)$ - $h$ factorization [Fig. 2(c)]

$$
\Sigma_{\alpha, \beta}^{(0), G^{(2)}}=\sum_{m}^{\mathcal{D}^{\mathrm{N}+2}} \sum_{q}^{\mathcal{D}^{\mathrm{N}-1}}\langle\alpha q \mid m\rangle\langle m \mid q \beta\rangle+(h-h)-p \text { terms, }
$$

TABLE I. Parameters of CI calculations for the $\mathrm{Na}_{9}{ }^{+}$cluster. The active space comprises 12 orbitals, four of them are doubly occupied in the ground state. Excited states are generated by allowing at most $n_{h}$ holes in the initially occupied orbitals and at most $n_{e}$ electrons in the initially unoccupied orbitals. $\varkappa$ denotes the multiplicity of states. $\varepsilon_{1}$ is the energy of the first-excited state (the optical gap). $E_{E A}$ and $E_{E A}^{I I}$

\begin{tabular}{|c|c|c|c|c|c|c|c|c|c|c|}
\hline$\varkappa$ & $\mathcal{D}^{\mathrm{N}}$ & $\mathcal{D}^{\mathrm{N}+1}$ & $\mathcal{D}^{\mathrm{N}-1}$ & $\mathcal{D}^{\mathrm{N}+2}$ & $\mathcal{D}^{\mathrm{N}-2}$ & $\varepsilon_{1}$ & $E_{E A}$ & $E_{I P}$ & $E_{E A}^{I I}$ & $E_{I P}^{I I}$ \\
\hline \multicolumn{11}{|c|}{$n_{h}=n_{e}=1$} \\
\hline & 65 & 8 & 4 & 64 & 16 & & & & & \\
\hline 1 & 33 & & & 36 & 10 & 1.70 & & & 2.42 & 17.56 \\
\hline 2 & & 8 & 4 & & & & 2.57 & 7.00 & & \\
\hline 3 & & & & 28 & 6 & & & & 2.36 & 17.30 \\
\hline \multicolumn{11}{|c|}{$n_{h}=n_{e}=2$} \\
\hline$*$ & 1425 & 376 & 180 & 1856 & 400 & & & & & \\
\hline 1 & 561 & & & 708 & 170 & 2.02 & & & 2.41 & 17.49 \\
\hline 2 & & 264 & 132 & & & & 2.14 & 7.32 & & \\
\hline 3 & & & & 924 & 198 & & & & 2.38 & 17.19 \\
\hline \multicolumn{11}{|c|}{$n_{h}=n_{e}=3$} \\
\hline$*$ & 12625 & 5640 & 2500 & 19776 & 3600 & & & & & \\
\hline 1 & 4145 & & & 6336 & 1310 & 1.66 & & & 2.96 & 17.28 \\
\hline 2 & & 3288 & 1524 & & & & 2.75 & 6.94 & & \\
\hline 3 & & & & 9408 & 1746 & & & & 2.91 & 16.97 \\
\hline \multicolumn{11}{|c|}{$n_{h}=n_{e}=4$} \\
\hline & 55325 & 37280 & 15100 & 99520 & 14800 & & & & & \\
\hline 1 & 16605 & & & & & 1.65 & & & & \\
\hline 2 & & 19360 & 8300 & & & & 2.77 & 6.92 & & \\
\hline
\end{tabular}
are the single- and double-electron affinities, whereas $E_{I P}$ and $E_{I P}^{I I}$ are the single- and double-ionization potentials, respectively. Energies are given in $\mathrm{eV}$. The CISDTQ calculations $\left(n_{h}=n_{e}=4\right)$ could not have been performed for all configurations due to memory limitations. 


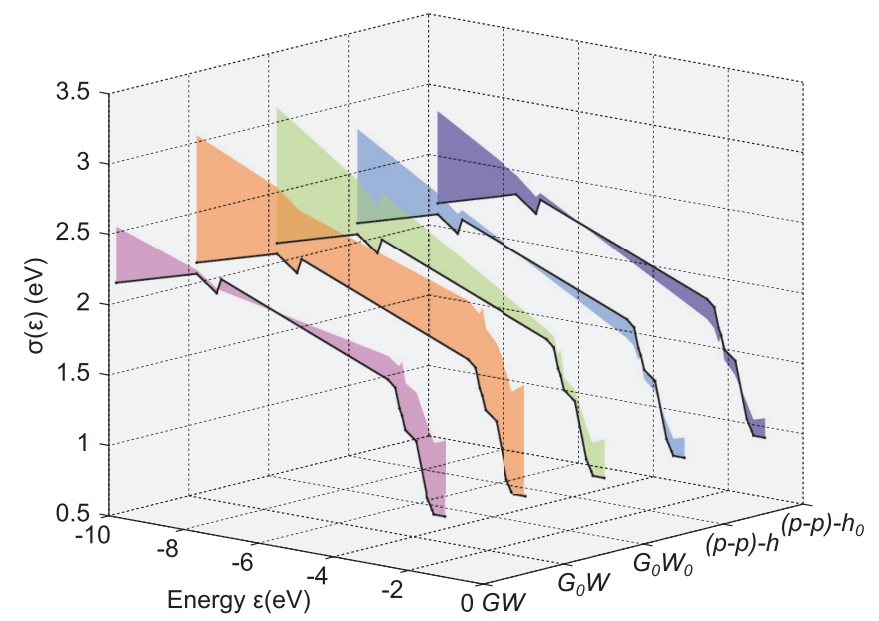

FIG. 3. (Color online) Energy uncertainty [Eq. (12)] of the valence states of $\mathrm{Na}_{9}{ }^{+}$cluster computed at different levels of theory. Shaded area denotes deviation of the approximate theories from the exact.

where similar to Eq. (17) we define the convolution of $\langle\alpha \beta \mid \gamma \delta\rangle$ with the matrix elements of the two creation (annihilation) operators $P_{\gamma \delta}^{m}=\left\langle m \mathrm{~N}+2\left|\hat{a}_{\gamma}^{\dagger} \hat{a}_{\delta}^{\dagger}\right| 0 \mathrm{~N}\right\rangle$ :

$$
\langle\alpha q \mid m\rangle=\sum_{\beta} \sum_{\gamma \delta} Y_{\beta}^{q}\langle\alpha \beta \mid \gamma \delta\rangle P_{\gamma \delta}^{m} .
$$

The $(p-p)-h$ factorization of the $2 p 1 h$ Green's function is nothing but the well-known ladder-diagram expansion. Equations (16) and (18) can be thought of as the Fermi golden rule expressions. Since the delta-function ensuring energy conservation is not present here the whole expression has the dimensions of energy squared. Starting from Eqs. (16) and (18) we further derive a series of simpler approximations. When the HF Green's function is used in place of $\mathcal{G}$ we obtain the so-called $G^{0} W$ approximation. If, furthermore, the noninteracting excited states are used to compute $Q_{\gamma \delta}^{n}$ we obtain the $G^{0} W^{0}$ approximation with the spectral moment:

$$
\Sigma_{\alpha, \beta}^{(0), G^{0} W^{0}}=2 \sum_{\eta, \gamma, \delta}\langle\alpha \eta \mid \gamma \delta\rangle\langle\gamma \delta \mid \eta \beta\rangle n_{\gamma}\left(1-n_{\delta}\right),
$$

where $n_{\gamma}$ is the occupation of the single-particle state $\gamma$. This is a rather simple and accurate approach as the comparison
(Fig. 4) shows. Calculations on this level are feasible even for much larger systems (such as $\mathrm{C}_{60}$ fullerene $^{36}$ ).

For the $\mathrm{Na}_{9}{ }^{+}$cluster we computed the spectral moments [Eqs. (14), (16), and (18)] by exact diagonalization of the many-body Hamiltonian. The details of CI calculations are presented in Table I. Comparison of CI singles, doubles, and triples (CISDT) and CI singles, doubles, triples, and quadruples (CISDTQ) calculations allows us to estimate the accuracy of the former method to be in the range of $20 \mathrm{meV}$ which is sufficient for the present discussion. In what follows we only report the CISDT results. The exact spectral function [solid line, Fig. 4(a)] of the lowest valence state $\left(\varepsilon_{1}^{\mathrm{HF}}=-9.786 \mathrm{eV}\right)$ is fragmented (two major peaks) and has multiple satellites. Despite this fact the model spectral function centered at the HF energy approaches the exact one in a large range of energies. The exact energy uncertainty from the first two spectral moments of the spectral function [Eq. (14)] is compared with the expressions resulting from the approximation for the self-energy [Eqs. (20), (16), and (18)] (Fig. 3). Corresponding self-energy spectral functions and weights are shown in Figs. 4(b) and 4(c). Generally, the $G W$ self-energy yields results superior to other approximations studied here. Equally surprising is the large energy gap in the ladder approximation. It is possible, however, to relate this effect to the large magnitude of the second-ionization potentials (last column of Table I). To make a quantitative statement we derive expressions for the gap in the self-energy spectrum as

$$
\begin{aligned}
& \Delta_{(p-h)-p}=E_{I P}-E_{E A}+2 \varepsilon_{1}=\Delta^{N}+2 \varepsilon_{1}, \\
& \Delta_{(p-p)-h}=E_{I P}^{I I}-E_{E A}^{I I}+E_{I P}-E_{E A}=\Delta^{N}+E_{I P}^{I I}-E_{E A}^{I I},
\end{aligned}
$$

where $\Delta^{N}$ is the quasiparticle gap. It is obvious now that the difference in gaps of the two self-energies arises due to the electron correlations: only for noninteracting systems does the double energy of the first-excited state equal $E_{I P}^{I I}-E_{E A}^{I I}$; for the gapped system they typically lead to $E_{I P}^{I I}-E_{E A}^{I I} \gg 2 \varepsilon_{1}$. This is an extension to two-particle case of a fact well known in quantum chemistry that $\Delta^{N} \gg \varepsilon_{1}$ (positivity of the exciton and biexciton binding energies).

Despite the large deviation in energy positions and, therefore in the shape of the self-energy spectral function $\mathbf{S}(\omega)$ [cf. solid and dash-dotted lines in Fig. 4(c)] the spectral moments are rather accurate and are close to those of $G^{0} W^{0}$

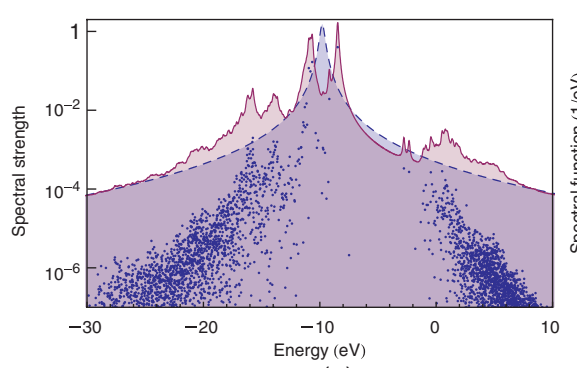

(a)

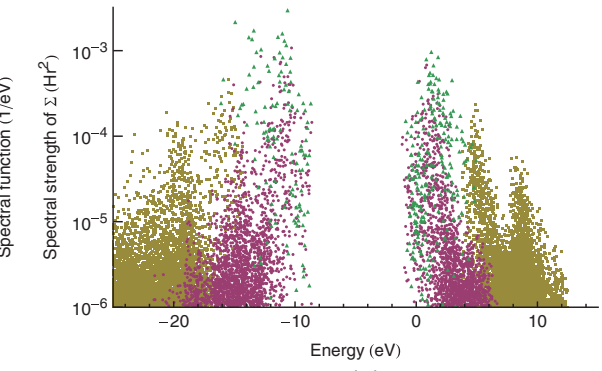

(b)

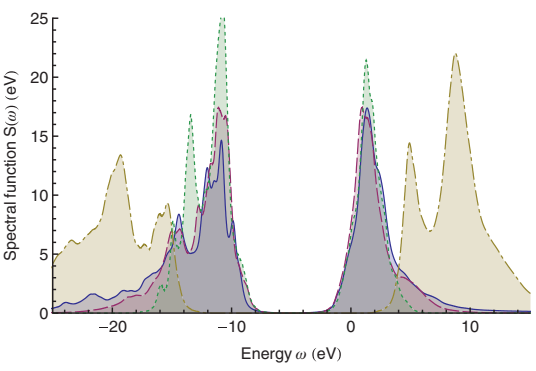

(c)

FIG. 4. (Color online) CI calculations for the $\mathrm{Na}_{9}{ }^{+}$cluster. (a) The exact (solid) and model (dashed) spectral functions of the lowest valence state. The dots denote the weights [squares of the matrix elements, Eq. (15)] of the exact spectral function. (b) The spectral strength of the self-energy in ( $p$ - $h$ )- $p$ (red dots) and $(p-p)$ - $h$ (yellow squares) factorizations; green triangles denote the $G^{0} W^{0}$ results. (c) The spectral function $[\operatorname{Tr}[\mathbf{S}(\omega)]]$ of the exact (solid), $(p-h)-p$ (dashed), $(p-p)-h$ (dash-dotted), and $G^{0} W^{0}$ (dotted) self-energies. 
approximation. The latter approximation, albeit its simplicity, closely approaches the exact self-energy in the vicinity of the gap between the highest-occupied molecular orbital and the lowest-unoccupied molecular orbital (HOMO-LUMO gap). For higher and lower energies the agreement, however, becomes poor and it confirms a well-known fact that the satellite structures cannot be reproduced within this approach. ${ }^{37}$

\section{CONCLUSIONS}

Summarizing, we presented a form of the quasiparticle lineshape that reflects the correct short- and long-time limits of the single-particle Green function and, thus, can be used via, e.g., the generalized Kadanoff-Baym ansatz ${ }^{38,39}$ to parametrize electronic structure that evolve in time (e.g., attosecond time-resolved photoemission). En route, we made some assumptions allowing us to construct the spectral function that is physically valid in the whole energy range with the correct asymptotic behavior. These are as follows: for a given particle-hole excitation the spectral function is represented by a single peak positioned at the mean-field energy having the strength exactly equal to one to ensure the normalization. In contrast, in the theory of Fermi liquids one is focused on a small interval of energies around the quasiparticle peak (shifted from the mean-field position) where the shape is essentially Lorentzian with a spectral weight $Z<1$. The normalization is restored by adding a smooth incoherent background. If even a more accurate description of the peak is desired the asymmetric Breit-Wigner form can be derived. ${ }^{40}$ This, however, further reduces the admissible range of energies because in the vicinity of the peak the spectral density already becomes negative. Our interpolative formula certainly misses such detailed structure in the energy domain having put more emphasis on the time evolution.

The main ingredient of our approach is the energy uncertainty $\sigma^{2}(\epsilon)$ of many-body states. The CI method enables us to compute it either exactly or by a number of diagrammatic approximations. Such calculations, besides providing an input for the description of the state's time evolution, also give a measure of the approximations' actual accuracy. Our simulations indicate that for the system considered here rather accurate results are obtained by neglecting the three-particle correlations. Among the possible decoupling schemes: the $G W$ approach $[(p-h)-p]$ and the ladder approximation $[(p-p)-h]$ the former gives more accurate results, although this might not hold in general. Regarding experiments measuring the initial time of the decay, we note that that this decay will generally be quadratic in time. The important quantity, however, is the decay constant $\sigma$ which can be easily extracted experimentally from the initial time evolution. As evident from Fig. 3, various levels of approximation and/or different contributing processes lead to a different $\sigma$ that can be considered as the material-dependent quantity to be determined in an attosecond time-resolved experiment for the decaying excitations.

\section{ACKNOWLEDGMENTS}

The work is supported by DFG-SFB762 (Y. P., J. B.). A. R. acknowledge financial support from the European Research Council Advanced Grant DYNamo (ERC-2010-AdG-
Proposal No. 267374), Spanish Grants (FIS2011-65702 C0201 and PIB2010US-00652), ACI-Promociona (ACI20091036), Grupos Consolidado UPV/EHU del Gobierno Vasco (IT-319-07), and European Commission projects CRONOS (280879-2 CRONOS CP-FP7) and THEMA (FP7-NMP-2008SMALL-2, 228539). Computational time was granted by i2basque and BSC Red Espanola de Supercomputacion.

\section{APPENDIX A: PROPERTIES OF SPECTRAL FUNCTION}

a. Definitions. We can start from the Lehmann representation

$$
\mathcal{G}_{i j}(\omega)=\sum_{\mu \in \mathcal{D}^{\mathrm{N}+1}} \frac{\mathcal{A}_{\mu_{i j}}^{+}}{\omega-\epsilon_{\mu}^{+}+i \eta}+\sum_{\mu \in \mathcal{D}^{\mathrm{N}-1}} \frac{\mathcal{A}_{\mu}^{-}}{\omega-\epsilon_{\mu}^{-}-i \eta},
$$

where

$$
\begin{aligned}
& \epsilon_{\mu}^{+}=E_{\mu}^{(N+1)}-E_{0}^{(N)} \geqslant-E_{E A}, \\
& \epsilon_{\mu}^{-}=E_{0}^{(N)}-E_{\mu}^{(N-1)} \leqslant-E_{I P} .
\end{aligned}
$$

In terms of previously introduced matrix elements [Eq. (15)] the residues of the Green's function are given by

$$
\begin{aligned}
& A_{\mu_{i j}}^{+}=\left\langle 0 \mathrm{~N}\left|\hat{a}_{i}\right| \mu \mathrm{N}+1\right\rangle\left\langle\mu \mathrm{N}+1\left|\hat{a}_{j}^{\dagger}\right| 0 \mathrm{~N}\right\rangle, \\
& A_{\mu_{i j}}^{-}=\left\langle 0 \mathrm{~N}\left|\hat{a}_{i}^{\dagger}\right| \mu \mathrm{N}-1\right\rangle\left\langle\mu \mathrm{N}-1\left|\hat{a}_{j}\right| 0 \mathrm{~N}\right\rangle .
\end{aligned}
$$

We apply the identity

$$
\frac{1}{x \pm i \eta}=\mathcal{P} \frac{1}{x} \mp i \pi \delta(x)
$$

and obtain

$$
\begin{aligned}
\frac{1}{\pi} \operatorname{Im} \mathcal{G}_{i j}(\omega)= & -\sum_{\mu \in \mathcal{D}^{\mathrm{N}+1}} \mathcal{A}_{\mu}^{+} \delta\left(\omega-\epsilon_{\mu}^{+}\right) \\
& +\sum_{\mu \in \mathcal{D}^{\mathrm{N}-1}} \mathcal{A}_{\mu}^{-} \delta\left(\omega-\epsilon_{\mu}^{-}\right) .
\end{aligned}
$$

Typically, and we will always assume it, there is a well-defined Fermi level $\epsilon_{F}$ located somewhere between the negative of the ionization potential $\left(\sim \epsilon_{\mathrm{HOMO}}\right)$ and of the electron affinity $\left(\sim \epsilon_{\text {LUMO }}\right)$

$$
\epsilon_{\mu}^{-} \leqslant-E_{I P} \leqslant \epsilon_{F} \leqslant-E_{E A} \leqslant \epsilon_{\mu}^{+} .
$$

In fact, some examples to demonstrate a possibility of $-E_{E A}<$ $-E_{I P}$ have been constructed. ${ }^{41}$ There, the authors consider the density functional theory with continuous number of electrons $N$ and construct the total energy which is concave as a function of $N$. This implies the negative hardness $\eta$ defined as

$$
\eta=\frac{1}{2} \frac{\partial^{2} E}{\partial N^{2}} \stackrel{N \in \mathbb{N}}{=} \frac{E_{I P}-E_{E A}}{2} .
$$

For the usual case (A3) we can introduce the spectral representation

$$
\mathcal{G}_{i j}(\omega)=\int_{C} \frac{S_{i j}\left(\omega^{\prime}\right)}{\omega-\omega^{\prime}} d \omega^{\prime},
$$

where the contour runs infinitesimally close from above to the real axis for energies below the Fermi energy and below the 
real axis for energies above the Fermi energy (see Fig. 3 of Ref. 42). It follows then

$$
\begin{aligned}
S_{i j}(\omega) & =\sum_{\mu \in \mathcal{D}^{\mathrm{N}+1}} \mathcal{A}_{\mu}^{+} \delta\left(\omega-\epsilon_{\mu}^{+}\right)+\sum_{\mu \in \mathcal{D}^{\mathrm{N}-1}} \mathcal{A}_{\mu}^{-} \delta\left(\omega-\epsilon_{\mu}^{-}\right) \\
& = \pm \frac{1}{\pi} \operatorname{Im} \mathcal{G}_{i j}(\omega),
\end{aligned}
$$

where ( + ) stands for $\omega<\epsilon_{F}$ and (-) for $\omega>\epsilon_{F}$. From the last expression, Eq. (14) follows trivially.

b. Relation between properties of $\mathcal{G}$ and $S$ at small times. It is reasonable to assume the following general expansion of the electron Green's function:

$$
\mathcal{G}_{k}(t)=\mathcal{G}_{k}^{0}(t) \sum_{n} a_{n}(i t)^{n} .
$$

This can most naturally come from the cumulant expansion. What is the corresponding spectral function? In the $\omega$ space it is given by

$$
S_{k}(\omega)=\sum_{n} a_{n} \delta^{(n)}\left(\omega-\epsilon_{k}\right)
$$

where $\delta^{(n)}(\omega)$ formally denotes the $n$th derivative of the $\delta$ function. Henceforth, after the Fourier transform we obtain

$$
S_{k}(t)=\sum_{n} a_{n} \int \frac{d \omega}{2 \pi} e^{-i \omega t} \delta^{(n)}\left(\omega-\epsilon_{k}\right)=\frac{e^{-i \epsilon_{k} t}}{2 \pi} \sum_{n} a_{n}(i t)^{n} .
$$

\section{APPENDIX B: DERIVATION OF SELF-ENERGY REPRESENTATION IN TERMS of $2 p 1 h$ IRREDUCIBLE GREEN'S FUNCTION}

We start with the equations of motion for the general oneand two-particle Green's function. We do not specify from the beginning what channel $p$ - $h$ or $p$ - $p$ we consider. It will be clear later when we introduce a specific ordering of the time arguments.

$$
\mathcal{G}\left(1,1^{\prime}\right)=\mathcal{G}^{0}\left(1,1^{\prime}\right)-i \int d(23) \mathcal{G}^{0}(1,2) v(23) \mathcal{G}\left(23,3^{+} 1^{\prime}\right),
$$

with

$$
(i) \equiv\left(\mathbf{r}_{i}, s_{i}, t_{i}\right), \quad\left(i^{+}\right) \equiv\left(\mathbf{r}_{i}, s_{i}, t_{i}+\delta\right), \quad \delta>0 .
$$

By comparing with the Dyson equation we see that the selfenergy can be written in terms of the two-particle Green's function:

$$
\Sigma\left(1,1^{\prime}\right)=-i \int d(23) v(13) \mathcal{G}\left(13,3^{+} 2\right) \mathcal{G}^{-1}\left(21^{\prime}\right) .
$$

Thus, we will try to express the self-energy in terms of a higher-order correlation function:

$$
\begin{aligned}
\mathcal{G}\left(12,1^{\prime} 2^{\prime}\right)= & \mathcal{G}^{0}\left(1,2^{\prime}\right) \mathcal{G}\left(2,1^{\prime}\right)-\mathcal{G}^{0}\left(1,1^{\prime}\right) \mathcal{G}\left(2,2^{\prime}\right) \\
& -i \int d(34) \mathcal{G}^{0}(1,3) v(34) \mathcal{G}\left(324,1^{\prime} 2^{\prime} 4^{+}\right)
\end{aligned}
$$

However, we must eliminate $\mathcal{G}^{0}$ from (B3). To do so we take the inverse of (B1):

$$
\begin{aligned}
& {\left[\mathcal{G}^{0}\right]^{-1}\left(1,1^{\prime}\right)} \\
& \quad=\mathcal{G}^{-1}\left(1,1^{\prime}\right)-i \int d(23) v(12) \mathcal{G}\left(12,2^{+} 3\right) \mathcal{G}^{-1}\left(3,1^{\prime}\right),
\end{aligned}
$$

multiply (B3) by $\mathcal{G}(6,5)\left[\mathcal{G}^{0}\right]^{-1}(5,1)$ from the left (the side actually is not important as the functions are commutative) and integrate:

$$
\begin{aligned}
\int & d(51) \mathcal{G}(6,5)\left[\mathcal{G}^{0}\right]^{-1}(5,1) \mathcal{G}\left(12,1^{\prime} 2^{\prime}\right) \\
= & \mathcal{G}\left(6,2^{\prime}\right) \mathcal{G}\left(2,1^{\prime}\right)-\mathcal{G}\left(6,1^{\prime}\right) \mathcal{G}\left(2,2^{\prime}\right) \\
& -i \int d(34) \mathcal{G}(6,3) v(34) \mathcal{G}\left(324,1^{\prime} 2^{\prime} 4^{+}\right),
\end{aligned}
$$

and finally use (B4) on the left-hand side of (B5). A rather long expression follows:

$$
\begin{aligned}
& \mathcal{G}\left(62,1^{\prime} 2^{\prime}\right)-i \int d(7341) \mathcal{G}(6,3) v(34) \mathcal{G}\left(34,4^{+} 7\right) \mathcal{G}^{-1}(7,1) \\
& \quad \times \mathcal{G}\left(12,1^{\prime} 2^{\prime}\right)=\mathcal{G}\left(6,2^{\prime}\right) \mathcal{G}\left(2,1^{\prime}\right)-\mathcal{G}\left(6,1^{\prime}\right) \mathcal{G}\left(2,2^{\prime}\right) \\
& -i \int d(34) \mathcal{G}(6,3) v(34) \mathcal{G}\left(324,1^{\prime} 2^{\prime} 4^{+}\right) .
\end{aligned}
$$

Here we introduce the irreducible function $G^{(3)}$ according to

$$
\begin{aligned}
& G^{(3)}\left(123,1^{\prime} 2^{\prime} 3^{\prime}\right)=\mathcal{G}\left(123,1^{\prime} 2^{\prime} 3^{\prime}\right) \\
& \quad-\int d(45) \mathcal{G}\left(13,3^{\prime} 4\right) \mathcal{G}^{-1}(4,5) \mathcal{G}\left(52,1^{\prime} 2^{\prime}\right)
\end{aligned}
$$

and write, after the reordering of variables,

$$
\begin{gathered}
\mathcal{G}\left(12,1^{\prime} 2^{\prime}\right)=\mathcal{G}\left(1,2^{\prime}\right) \mathcal{G}\left(2,1^{\prime}\right)-\mathcal{G}\left(1,1^{\prime}\right) \mathcal{G}\left(2,2^{\prime}\right) \\
-i \int d(34) \mathcal{G}(1,3) v(34) G^{(3)}\left(324,1^{\prime} 2^{\prime} 4^{+}\right) .
\end{gathered}
$$

Now we can substitute it in the definition (B2). After trivial but lengthy manipulation we obtain

$$
\begin{aligned}
\Sigma\left(1,1^{\prime}\right)= & \delta\left(11^{\prime}\right) \int d(3) n(3) v(13)-v\left(11^{\prime}\right) n\left(11^{\prime}\right) \\
& +\int d(36) v(13) G^{(3)}\left(6^{+} 13,63^{+} 1^{\prime}\right) v\left(61^{\prime}\right) .
\end{aligned}
$$

The first term here is just the direct interaction with the usual definition of the density $n(1)=-i \mathcal{G}\left(1,1^{+}\right)$. The second term is the exchange interaction with the density matrix defined as $n\left(11^{\prime}\right)=-i \mathcal{G}\left(1,1^{\prime+}\right)$. These two terms are included in the mean-field Hamiltonian $\mathcal{H}^{0}\left(1,1^{\prime}\right)=\delta\left(11^{\prime}\right)[\mathcal{T}(1)+\mathcal{V}(1)]+$ $\Sigma_{\infty}^{0}\left(11^{\prime}\right)$ and are therefore excluded from the otherwise identical equation (14). The summation over the spin variables (included in the definition of the integrals) leads to the appearance of the prefactor 2 in the direct term for closedshell systems. The exact frequency-independent part of the self-energy is given by

$$
\Sigma_{\infty}\left(11^{\prime}\right)=\delta\left(11^{\prime}\right) \int d(3) n(3) v(13)-v\left(11^{\prime}\right) n\left(11^{\prime}\right),
$$

whereas in the mean-field Hamiltonian $\Sigma_{\infty}^{0}\left(11^{\prime}\right)$ is computed by the same formula from the zeroth-order densities, i.e., $n^{0}\left(11^{\prime}\right)=-i \mathcal{G}^{0}\left(1,1^{\prime+}\right)$. 
*yaroslav.pavlyukh@physik.uni-halle.de

${ }^{1}$ R. Cerbino, L. Peverini, M. A. C. Potenza, A. Robert, P. Bösecke, and M. Giglio, Nat. Phys. 4, 238 (2008).

${ }^{2}$ I. A. Walmsley, Science 319, 1211 (2008).

${ }^{3}$ F. Krausz and M. Ivanov, Rev. Mod. Phys. 81, 163 (2009).

${ }^{4}$ A. Cavalieri et al., Nature (London) 429, 1029 (2007).

${ }^{5}$ M. Schultze, M. Fieß, N. Karpowicz, J. Gagnon, M. Korbman, M. Hofstetter, S. Neppl, A. L. Cavalieri, Y. Komninos, T. Mercouris, C. A. Nicolaides, R. Pazourek, S. Nagele, J. Feist, J. Burgdörfer, A. M. Azzeer, R. Ernstorfer, R. Kienberger, U. Kleineberg, E. Goulielmakis, F. Krausz, and V. S. Yakovlev, Science 328, 1658 (2010).

${ }^{6}$ Collected Papers of L. D. Landau, edited by D. ter Haar (Gordon and Breach Science Publishers, New York, 1965), Chaps. 90 and 91.

${ }^{7}$ P. Nozières and D. Pines, The Theory of Quantum Liquids (Westview Press, Boulder, CO, 1999), p. 309.

${ }^{8}$ M. Vogt, R. Zimmermann, and R. J. Needs, Phys. Rev. B 69, 045113 (2004).

${ }^{9}$ M. Bonitz, D. Semkat, and H. Haug, Eur. Phys. J. B 9, 309 (1999).

${ }^{10}$ H. Haug and L. Bányai, Sol. State. Commun. 100, 303 (1996)

${ }^{11}$ B. Gumhalter, Phys. Rev. B 72, 165406 (2005).

${ }^{12}$ Y. Pavlyukh, A. Rubio, and J. Berakdar, arXiv:1212.3486.

${ }^{13}$ K. S. Thygesen and A. Rubio, Phys. Rev. B 77, 115333 (2008).

${ }^{14}$ K. Balzer, M. Bonitz, R. van Leeuwen, A. Stan, and N. E. Dahlen, Phys. Rev. B 79, 245306 (2009).

${ }^{15}$ M. Puig von Friesen, C. Verdozzi, and C.-O. Almbladh, Phys. Rev. Lett. 103, 176404 (2009).

${ }^{16}$ A typical approach to establish the positivity of a Fourier transform is to use the convexity of the function. Obviously, in our case the function is not convex everywhere, but this can be circumvented by shifting the contour of integration to the region where this condition is fulfilled.

${ }^{17}$ E. O. Tuck, Bull. Austr. Math. Soc. 42, 133 (2006).

${ }^{18}$ B. L. Altshuler, Y. Gefen, A. Kamenev, and L. S. Levitov, Phys. Rev. Lett. 78, 2803 (1997).
${ }^{19}$ J. Schirmer, L. S. Cederbaum, and O. Walter, Phys. Rev. A 28, 1237 (1983).

${ }^{20}$ M. Altarelli, D. L. Dexter, H. M. Nussenzveig, and D. Y. Smith, Phys. Rev. B 6, 4502 (1972).

${ }^{21}$ J. Winter, Nucl. Phys. A 194, 535 (1972).

${ }^{22}$ C. Barbieri, D. Van Neck, and W. H. Dickhoff, Phys. Rev. A 76, 052503 (2007).

${ }^{23}$ Y. Pavlyukh and W. Hübner, Eur. Phys. J. D 21, 239 (2002).

${ }^{24}$ Y. Pavlyukh and W. Hübner, Phys. Lett. A 327, 241 (2004).

${ }^{25}$ G. Pal, Y. Pavlyukh, H. C. Schneider, and W. Hübner, Eur. Phys. J. B 70, 483 (2009).

${ }^{26}$ Y. Pavlyukh and W. Hübner, Phys. Rev. B 75, 205129 (2007).

${ }^{27}$ Y. Pavlyukh, J. Berakdar, and W. Hübner, Phys. Rev. Lett. 100, 116103 (2008)

${ }^{28}$ J. Olsen, B. Roos, P. Jorgensen, and H. Jensen, J. Chem. Phys. 89, 2185 (1988).

${ }^{29}$ P. Knowles and N. Handy, Chem. Phys. Lett. 111, 315 (1984).

${ }^{30}$ R. Pauncz, Spin Eigenfunctions (Plenum, New York, 1979).

${ }^{31}$ G. Onida, L. Reining, and A. Rubio, Rev. Mod. Phys. 74, 601 (2002).

${ }^{32}$ C.-O. Almbladh, U. von Barth, and R. van Leeuwen, Int. J. Mod. Phys. B 13, 535 (1999).

${ }^{33}$ L. Hedin, Phys. Rev. 139, A796 (1965).

${ }^{34}$ D. H. Kobe, Ann. Phys. (NY) 19, 448 (1962)

${ }^{35}$ A. Barth and L. S. Cederbaum, Phys. Rev. A 23, 1038 (1981).

${ }^{36}$ Y. Pavlyukh and J. Berakdar, J. Chem. Phys. 135, 201103 (2011).

${ }^{37}$ M. Guzzo, G. Lani, F. Sottile, P. Romaniello, M. Gatti, J. J. Kas, J. J. Rehr, M. G. Silly, F. Sirotti, and L. Reining, Phys. Rev. Lett. 107, 166401 (2011).

${ }^{38}$ P. Lipavský, V. Špička, and B. Velický, Phys. Rev. B 34, 6933 (1986).

${ }^{39}$ T. Kita, Prog. Theor. Phys. 123, 581 (2010).

${ }^{40}$ F. Aryasetiawan, L. Hedin, and K. Karlsson, Phys. Rev. Lett. 77, 2268 (1996).

${ }^{41}$ M. H. Cohen and A. Wasserman, Isr. J. Chem. 43, 219 (2003).

${ }^{42}$ L. Hedin and S. Lundqvist, in Solid State Physics, Vol. 23, edited by D. T. Frederick Seitz and H. Ehrenreich (Academic Press, New York, 1970), pp. 1-181. 